\title{
Measurement of the Longitudinal Structure Function from ep Collisions with the H1 Detector at HERA
}

\author{
N. Raičević \\ on behalf of the $\mathrm{H} 1$ collaboration
}

Faculty of Science, University of Montenegro, Cetinjski put BB, 81000 Podgorica, Montenegro

\begin{abstract}
The longitudinal structure function, $F_{L}$, is identically zero in lowest order QCD, but due to gluon radiation gets a non-zero value in perturbative QCD. Measurements of $F_{L}$ can thus provide constraints on the gluon density in the proton which are complementary to that obtained from the scaling violations of structure function $F_{2}$. In this report indirect measurements of $F_{L}$ from $e p$ collisions with the $\mathrm{H} 1$ detector at HERA accelerator, at center-of-mass energy of $320 \mathrm{GeV}$, are summarised. For the measurement of the $F_{L}$ it is essential to reach maximum possible values of inelasticity $y$. Experimental techniques which can provide precise measurements in this region will also be discussed.
\end{abstract}

Keywords: Longitudinal structure functions, High y, Low x, Low $Q^{2}$, Deep inelastic scattering (DIS), HERA, H1, Perturbative Quantum Chromodynamics (pQCD), Next to Leading-Order (NLO)

PACS: 13.60.-r

In pQCD which predicts the existence of gluons interacting with quarks, $F_{L}$ gets sizable contributions at high values of inelasticity, $y>0.6$, which is related to the modulus of the squared four-momentum transfer, $Q^{2}$, and the Bjorken scale variable, $x$, as $y=Q^{2} / s x$. At present, values of $F_{L}$ are obtained by indirect methods which depend on the NLO QCD fit to the H1 data at high $Q^{2}$ or exploit the shape of the cross section which at high $y$ is driven by the $y$-dependent kinematic factor and to a lesser extend by $F_{L}$ which is considered to be constant. The results for fixed $y=0.75$ of all current $\mathrm{H} 1$ data in the $Q^{2}$ range $0.75<Q^{2}<700 \mathrm{GeV}^{2}$ are obtained [1]. In such methods for $F_{L}$ determination one assumes $F_{2}$ to be known, or it is simultaneously determined with $F_{L}$ at low $x$ when only the cross section is measured. At low values of $x$, where non-perturbative effects become significant, gluon density which has only been indirectly determined is thus poorly constrained by data. World fits of the inclusive cross sections deviate in their relative contribution of sea quarks and gluons at low $x$ by about a factor of two [2]. The low $x$ region with large parton density is still not well understood and a direct measurement of $F_{L}$ would be of great importance for its understanding [2].

A direct determination of $F_{L}$ requires cross section values measured at different $y$ values for the same values of $x$ and $Q^{2}$. This could be achieved by reducing the centre of mass energies by performing dedicated runs at lower proton beam energies. Such run has been considered to be very essential to complete the HERA ep program, which is largely devoted to the understanding of a high density system of partons.

At low values of $Q^{2}$ one has $y \simeq 1-E_{e}^{\prime} / E_{e}$ where $E_{e}$ and $E_{e}^{\prime}$ are energies of incoming and outgoing lepton, respec- tively. Thus, the scattered electron has to be identified in a large background of hadrons originating mainly from photoproduction. To get a precise measurement of $F_{L}$ it is necessary to provide relevant detection techniques in the high $y$ region and also essential to understand the systematics well.

H1 provides excellent access to the high-y region. At $Q^{2}>10 \mathrm{GeV}^{2}$, cluster from the backward lead-fibre calorimeter (SpaCal) is linked to the track from the central tracking detectors (backward is in $\mathrm{H} 1$ convention direction of lepton beam). At $Q^{2}<10 \mathrm{GeV}^{2}$, cluster from SpaCal is linked to the track from the Backward Silicon Tracker (BST). From the charge assignment of tracks associated with the SpaCal cluster, the background has been subtracted statistically. The sample of candidates with charge opposite to that of incoming lepton has been taken to represent the background. This allows the energy range to be extended down to $E_{e}^{\prime}=3 \mathrm{GeV}$ corresponding to $y \simeq 0.9$.

The main sources of correlated errors are measurements of: the energy of the scattered electron $(2 \%$ at $3 \mathrm{GeV}$ to $0.3 \%$ at $30 \mathrm{GeV}$ ), the angle of the scattered electron $\left(0.2 \mathrm{mrad}\right.$ in $\mathrm{BST}$ and $1 \mathrm{mrad}$ at $\left.\Theta_{e}<165^{\circ}\right)$ and residual photoproduction background. Uncorrelated efficiencies come from electron identification, trigger, vertex, radiative corrections which and are on the level of $1 \%$.

\section{REFERENCES}

1. H1 Collaboration, Proceedings of the DIS'2005 Workshop, Madison, pp. 233-236 (2005).

2. M. Klein, Proceedings of the DIS'2004 Workshop, Štrbské Pleso, pp. 309-314 (2004). 